\title{
Near Infrared Spectroscopic Study of Brain Activity during Cognitive Conflicts on Facial Expressions
}

\author{
Munehide Nakagawa, Mie Matsui*, Masatoshi Katagiri, Takatoshi Hoshino \\ Department of Psychology, Graduate School of Medicine and Pharmaceutical Sciences, University of Toyama, Japan \\ *Corresponding author: mmatsui@las.u-toyama.ac.jp
}

Received May 04, 2015; Revised May 15, 2015; Accepted May 20, 2015

\begin{abstract}
The Stroop task has been typically used for measuring cognitive functions of inhibition and interference. However, this task has limited applications with young children, because reading ability is required to perform the task. Using a new, non-letter Stroop-like task named the 'happy-sad task,' in which participants are instructed to say 'happy' for a sad face and 'sad' for a happy face, we can assess differences in inhibition in participants from early childhood to adulthood. We investigated whether differences between the happy-sad task and the letter Stroop task could be observed in brain activation of healthy participants $(\mathrm{N}=30)$, by using near-infrared spectroscopy (NIRS) and skin conductance responses (SCR). We focused on the right and left anterior prefrontal cortex and frontal pole, which are known as centers for response inhibition and processing of emotions. We used region-of-interest analysis that approximately covered these regions and compared brain activation patterns between the two tasks. Results indicated that there was prefrontal activation during both tasks. Particularly, the incongruent condition of the happysad task resulted in greater activation than the letter Stroop task. In addition, SCR amplitude for the happy-sad task was greater than that for the letter Stroop task. These findings suggest that brain activity in the happy-sad task is associated with suppression of emotions and inhibition of behavior.
\end{abstract}

Keywords: happy-sad task, letter Stroop task, near-infrared spectroscopy, skin conductance response, anterior prefrontal cortex, inhibition

Cite This Article: Munehide Nakagawa, Mie Matsui, Masatoshi Katagiri, and Takatoshi Hoshino, "Near Infrared Spectroscopic Study of Brain Activity during Cognitive Conflicts on Facial Expressions." Research in Psychology and Behavioral Sciences, vol. 3, no. 2 (2015): 32-38. doi: 10.12691/rpbs-3-2-3.

\section{Introduction}

In order to facilitate goal-directed behaviors, operations must be conducted to sort out relevant from irrelevant information. Inhibiting irrelevant information and resolving interference between incoming sources of information is a prerequisite for pursuing internal and external goals. Mechanisms for performing these activities are commonly referred to as 'Cognitive control' mechanisms [1]. A typical task used for measuring cognitive functions of inhibition and interference is the Stroop task [2]. The traditional Stroop task depends on matching colors and letters. In this task, letters are printed in different foreground colors. Participants are required to read either the letters, or NAME the background color on which the letters are written. When naming the color of a conflicting stimulus (incongruent task: e.g. the letters GREEN displayed in red), a strong prepotent tendency has been observed to read the letters as "green," which competes with the response to the color "red" [3]. It has been shown that the reaction time (RT) in the incongruent task is slower than that in the congruent task (e.g. the letter RED displayed in red). This is known as 'the Stroop effect'.

Previous studies have reported that the Stroop effect could be observed in children of 7-8 years of age, and that the effect increases with reading ability [4,5]. These studies have indicated that reading ability is related to the performance of the Stroop task, and that the task has only limited effects with young children. The most widely used Stroop-like task developed for young children is the daynight task, on which they were asked to say "day" whenever a black card with the moon and stars appeared and to say "night" when shown a white card with a bright sun [6]. The day-night task is used for the measurement of interference control in young children between 3 and 7 years of age [7]. However, this task has a ceiling effect in children aged 6-7 years [6,7]. In order to solve this problem, Lagattuta rt al [8] designed a new, non-letter, Stroop-like task known as the 'happy-sad task.' In this task, the participant is instructed to say 'happy' for a sad face and 'sad' for a happy face. Using this task, inhibition can be assessed in early childhood and young adulthood without problematic floor or ceiling effects. Furthermore, the happy-sad task does not result in ceiling effects even in adults [8].

Previous studies of the Stroop task that have used functional magnetic resonance imaging (fMRI) and positron emission tomography (PET) have indicated activation in at least one of three main brain regions: the anterior cingulate cortex (ACC), parietal lobe, and lateral prefrontal cortex [9]. The ACC is known to be involved in evaluative processes when responding to incongruent 
stimuli, such as monitoring errors and response conflicts [10]. Parietal cortex is known to play a role in maintaining sustained attention during the Stroop task $[11,12]$. The role of the prefrontal cortex, especially inferior frontal gyrus (IFG) in the Stroop task has been reported in a number of studies [13]. The left-IFG activation is known to reflect processing selection and processing bias in the incongruent Stroop condition [14], whereas the right-IFG could be related to response inhibition [15]. However, the happy-sad task is a new task and the neural basis of processing in this task has yet to be explored.

Recently, near-infrared spectroscopy (NIRS) has also been used for investigating neural processing during the Stroop task [16-21]. NIRS is an optical method that noninvasively monitors the cerebral hemodynamics of oxygenated hemoglobin (oxy-Hb) and deoxygenetated hemoglobin (deoxy-Hb), by measuring changes in the attenuation of near infrared light passing through tissue [22]. NIRS is a non-invasive measure and allows participants to perform speech tasks in a natural and comfortable environment, without being confined to a small, restricted space. Findings using the NIRS regarding the regions of activation in the Stroop task have corroborated those of fMRI and PET studies.

The purpose of the present study was to determine whether there were differences in prefrontal cortex activation between the happy-sad task and the letter Stroop task, by using NIRS. Furthermore, to explore the influence of affective valence, we used skin conductance response (SCR), which measures electrical conductance between two points on the skin. SCR is widely used in research on emotions and physiological arousal, because the sweat glands are controlled by the sympathetic nervous system.

We predicted that processing in the happy-sad task would be related to activation of the prefrontal cortex, similar to the incongruent condition of the letter Stroop task. In addition, because of the emotional stimuli used in the happy-sad task, we predicted that the amplitude of the SCR in this task would be greater than that in the letter Stroop task.

\section{Methods}

\subsection{Participants}

Healthy participants recruited through the community $(\mathrm{N}=30,16$ women and 14 men; Mean age $=23.4, S D=$ 5.3, Age range 18-39 years) took part in the study. All participants were right-handed and had normal or corrected-to-normal vision. They completed the Japanese version of the National Adult Reading Test (JART [23]) that assesses IQ. The mean estimated IQ of participants was 109.0 ( $S D=5.3$, range 92-120). The subjective feeling of psychological fatigue in participants was assessed using the Visual-Analogue Scale (VAS) score [24]. Sleep duration in the previous night was also assessed. Previous studies have indicated that individuals with Schizotypal personality disorder (SPD) and schizotypal traits have difficulties in conflict detection and cognitive inhibition when performing the letter Stroop task $[25,26]$. Therefore, the present study assessed schizotypal traits using the Japanese version of the
Schizotypal Personality Questionnaire Brief (SPQ-B-J [27]) and all participants were within normal range.

In accordance with the Helsinki Declaration, written informed consent was obtained from each participant before conducting the study. The ethics committee of the University of Toyama had approved the study protocol in advance.

\subsection{Apparatus and Stimuli}

The experiment was conducted in a soundproof chamber, to reduce distractions. E-Prime software (Psychology Software Tools, Inc.) was used to control the stimulus presentation. In each trial, visual stimuli were presented on a 17-inch computer monitor.

A standardized set of facial expression photos (ATR facial expression database 99: ATR-Promotions) was used as stimuli. This database of facial expression photos comprises Japanese Happy, Sad, and Neutral faces (4 women and 6 men). Each fifth photo of 24 happy female faces, 13 sad female faces, 39 happy male faces, and 18 sad male faces was selected randomly. Thus, five happy female and male faces, and five sad female and male faces (total 20 photos) were selected. The letters in the letter Stroop task used Japanese red, blue, and green Hiragana script with a 180-point font. All stimuli were displayed on a black background at the center of the monitor (see Figure 1).

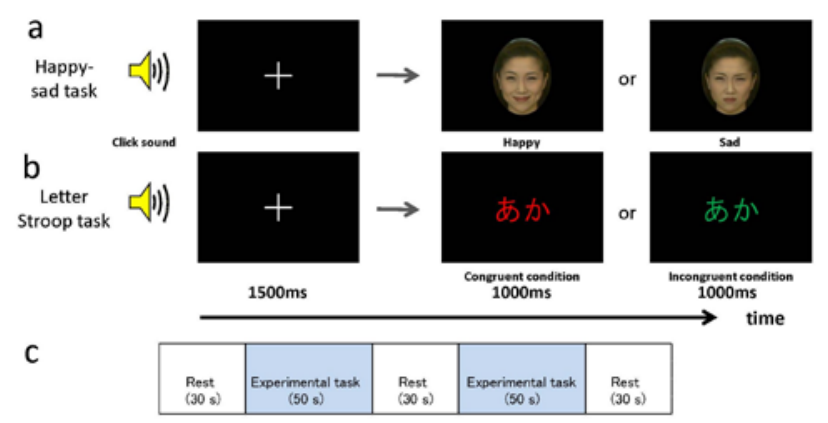

Figure 1. Sequence of the experimental trials. (a) Sequence of a trial in the happy-sad task. (b) Sequence of a trial in the letter Stroop task. (c) Each session contained an experimental task (each run 50 seconds) and a control task (resting period for 30 seconds per run) blocks

\subsection{Measurements}

A NIRS machine with 16 channels (OEG-16: Spectratech Inc., Yokohama, Japan) was used to measure the time courses of oxy-Hb and deoxy-Hb levels. Six emission and six detector probes were arranged in a 6 (width) $\times 2$ (length) matrix on the participants' forehead with an interoptode distance of $30 \mathrm{~mm}$. The center of the probe matrix was placed on Fpz (midpoint between Fp1 and Fp2 on the international 10-20 system for recording electroencephalograms). The NIRS system uses two wavelengths (approximately 770 and $840 \mathrm{~nm}$ ) of nearinfrared light. Absorption was recorded in order to estimate oxy-Hb levels. These signals were recorded simultaneously at a sampling rate of $1.54 \mathrm{~Hz}$ (see Figure 2).

The SCR was estimated at the distal phalanx of the left finger using a commercially available device (Toyo physical, Inc). For SCR recording, electrodes were attached to the forefinger and middle finger on the left hand. Data of the SCR were recorded simultaneously at a sampling rate of $10 \mathrm{~Hz}$. 


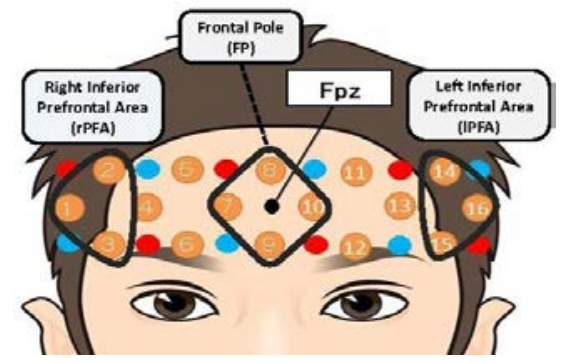

Figure 2. Schematic representation of near-infrared spectroscopy (NIRS) probes

The center of the probe matrix was placed on Fpz (midpoint between Fp1 and Fp2 on international 10-20 system for recording electroencephalograms). Red circles show illuminators and blue squares show detectors. Channels are presented as orange circles with channel numbers. Regions of interest (ROI) were right inferior prefrontal area (rPFA), frontal pole (FP), and left inferior prefrontal area (IPFA). Ch1-3 were labeled as rPFA, Ch7-10 were as FP, and Ch14-16 were as IPFA.

\subsection{Procedure}

NIRS and SCR measurements were conducted during resting periods of 30 seconds and when performing the congruent and incongruent conditions of the happy-sad and the letter Stroop task during 50 seconds. In the resting periods, a black background was presented on the monitor and participants were instructed to sit still with their eyes open. All conditions of both tasks were presented in a block design consisting of three phases (repetitions). Two blocks of congruent and incongruent presentation (one block $=20$ trials) separated by resting periods constituted one phase. Thus, both the happy-sad and the letter Stroop tasks were presented in 120 trials. The first phase of each experimental task started with the order: congruent followed by incongruent conditions and the next phase was conducted randomly. Before each experimental task, participants completed one practice phase (one block of 16 trials, and a total 32 trials).Participants were instructed to respond as accurately as possible (Figure 1).

\subsubsection{Happy-sad Task}

The happy-sad task consisted of a congruent and an incongruent condition. For congruent trials, participants were instructed to vocally state the facial expression that was displayed. For incongruent trials, participants were instructed to vocally state a different facial expression from the one that was displayed. Before starting, and after finishing the two experimental conditions, a clicking sound was presented as a warning stimulus to inform the participant of each opening and end of experimental conditions. In the congruent condition one click was presented, whereas two clicks were presented in the incongruent condition. Then, a fixation cross appeared for $1500 \mathrm{~ms}$, and a facial expression stimulus (a happy or sad face) was displayed for $1000 \mathrm{~ms}$. The two facial expression stimuli (total 20 photos) were presented in random order with equal probability.

After the end of the experiment, participants evaluated the affective valence of the facial expression stimuli used in the happy-sad task that consisted of 10 happy faces, 10 sad faces, and 10 neutral faces, presented randomly. Evaluation was conducted on a 7-point scale.

\subsubsection{Letter Stroop Task}

The letter Stroop task also consisted of a congruent and an incongruent condition. A color name was shown using letters of identical color to the color name (e. g., RED printed in red colored letters), for the congruent trial. A color name was printed using letters of a different color to the color name (e. g., RED printed in green colored letters) so as to produce an interference between the color of letters and color names, for the incongruent trial. Red, blue and green were used to print the color names. Participants were instructed to vocalize the color name in both conditions. In this task, one warning click was used before starting and after finishing in both conditions. A fixation cross appeared for $1500 \mathrm{~ms}$, and then a letter stimulus was displayed for $1000 \mathrm{~ms}$. The three color letters and color names were presented in random order with equal probability.

\subsection{Statistical Analyses}

\subsubsection{Behavioral Data Analyses}

In the happy-sad task and the letter Stroop task the analysis of the correct response ratio was conducted using the Wilcoxon signed-rank test. The affective valence of facial expressions was subjected to a one-way analysis of variance (ANOVA). Post hoc comparison was performed using Bonferroni correction.

\subsubsection{NIRS Data Analyses}

Changes in oxy-Hb and deoxy-Hb concentration were conducted as an index of changes in cerebral blood flow. Baseline correction was made using linear fitting, based on two sets of baseline data. Blocks affected by movement artifacts were removed. The remaining concentrations of oxy-Hb and deoxy-Hb during the 50-second task condition were then averaged. We used two-tailed one-sample $t$-tests to examine whether the hemodynamic response in the prefrontal areas was significantly different from baseline.

In this study, we analyzed NIRS data for the following measurement points that were labeled, Ch1-3 for right inferior prefrontal area (rPFA), Ch14-16 for left inferior prefrontal area (IPFA) and Ch7-10 for prefrontal pole (FP), approximately covering the right and left anterior prefrontal cortex and frontal pole, respectively (Okamoto et al., 2004). We focused on these channels as regions of interest (ROI) and conducted a two-way repeated measures ANOVA with the task (happy-sad task, letter Stroop task) and condition (congruent, incongruent) as within-subject factors for each ROI.

\subsubsection{SCR Data Analyses}

The SCR amplitudes were calculated by subtracting SCR value at the starting point of each task trial from the grand average SCR value for each task trial. Logtransformation $(\log [\mathrm{SCR}+1]$ was used to normalize SCR amplitude data [28]. We conducted two-way repeated measures ANOVA with tasks (happy-sad task, letter Stroop task) and condition (congruent, incongruent) as within-subject factors.

\section{Results}

\subsection{Behavioral Data}

The mean correct response ratio in each task was over $95 \%$ (happy-sad task: congruent condition, $99.2 \% \pm 1.3$; 
incongruent condition, $95.9 \% \pm 2.4$; letter Stroop task: congruent condition, $99.7 \% \pm 0.8$; incongruent condition, $98.7 \% \pm 1.4)$. The mean correct response ratio in the incongruent conditions of the happy-sad task and letter Stroop task was significantly lower than that in the congruent conditions of both tasks $(Z=-4.57, p<.001 ; Z$ $=-3.34, p<.05$, respectively). The congruent conditions were not significantly different between the two tasks $(Z=$ -1.60 , n.s.), whereas the incongruent conditions were significantly different $(Z=-4.40, p<.001)$.

Mean scores for the happy face stimuli, neutral face stimuli and sad face stimuli were $6.27 \pm 0.46,3.73 \pm 0.33$ and $1.56 \pm 0.42$, respectively. There was a significant main effect for affective valence of facial expression stimuli used in the happy-sad task $(F(2,28)=471.47, p<.001)$. Post hoc comparison indicated significant differences for all affective valences of facial expressions ( $p s<.001)$. Thus, the happy face stimuli were evaluated more positively than the neutral face stimuli. The sad face stimuli were evaluated more negatively than the neutral face stimuli.

Mean and SDs of SPQ-B-J was 6. 47 \pm 3.17 (range 0-14), that of VAS score was $48.07 \pm 21.35$ (range 6-86), sleep length was 6.12 \pm 1.49 hours (range 3-8 hour), and sleepiness was $3.17 \pm 1.15$ (Range 1-5). These results indicated that all scores of all participants were within the normal range.

\subsection{NIRS Data}

Changes in oxy-Hb concentration for each task and condition are presented in Figure 3. Changes in oxy-Hb concentrations in both tasks for the congruent condition were not significantly different in any ROIs. Change in oxy-Hb concentrations in both tasks for the incongruent condition was significantly different in all ROIs (happysad task, $p s<.01$; letter Stroop task, $p s<.05$ ).

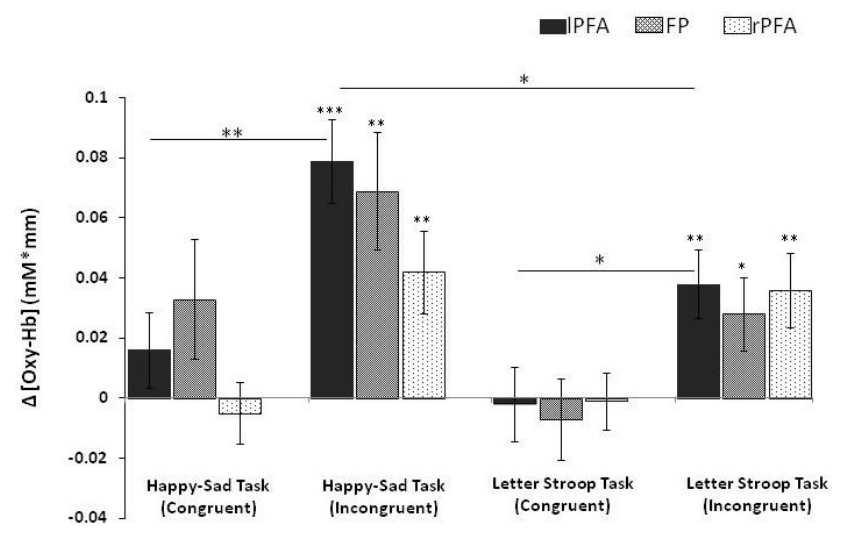

Figure 3. Change of oxy-Hb concentration for each task and condition in each ROI.

${ }^{*} \mathrm{p}<0.05, * * \mathrm{p}<0.01, * * * \mathrm{p}<0.001$.

In the IPFA, a two way ANOVA revealed a significant main effect of task $(F(1,28)=6.12, p<.05)$ and condition $(F(1,28)=37.14, p<.001)$. The interaction between task and condition was significant $(F(1,28)=$ 5.24, $p<.05$ ). The post hoc comparison was significant for each condition in both tasks (happy-sad task, $F(1,28)$ $=56.11, p=.001$; letter Stroop task, $F(1,28)=12.71, p$ $<.01)$. In the incongruent condition, there was a significant difference between tasks $(F(1,28)=9.60, p$ $<.01)$. In the FP, there were significant main effects of task $(F(1,28)=5.29, p<.05)$ and condition $(F(1,28)=$ 15.88, $p<.001)$. The interaction between task and condition was not significant. In the rPFA, there was a significant main effect of condition $(F(1,28)=20.49, p$ $<.001)$, whereas there was no significant main effect of task, or any significant interactions (Figure 3).

Change of deoxy-Hb concentration for each task and condition is presented in Figure 4. In the incongruent condition of the happy-sad task, there was a significant difference in the FP $(p<.05)$. In the congruent condition of the letter Stroop task, there were significant differences in the IPFA $(p<.01)$ and $\mathrm{FP}(p<.01)$. In the incongruent condition, there were significant differences in all ROIs $(p s<.05)$.

A two way ANOVA revealed that there was a main effect of task $(F(1,28)=8.45, p<.01)$ in the lPFA, there were no significant main effects of condition, nor a significant interaction between task and condition. In the FP, there were significant main effects of task $(F(1,28)=$ $10.20, p<.01)$ and condition $(F(1,28)=9.05, p<.01)$, whereas there was no interaction between task and condition. In the rPFA there was a significant main effect of condition $(F(1,28)=20.49, p<.001)$, whereas there was no main effect or any interactions.

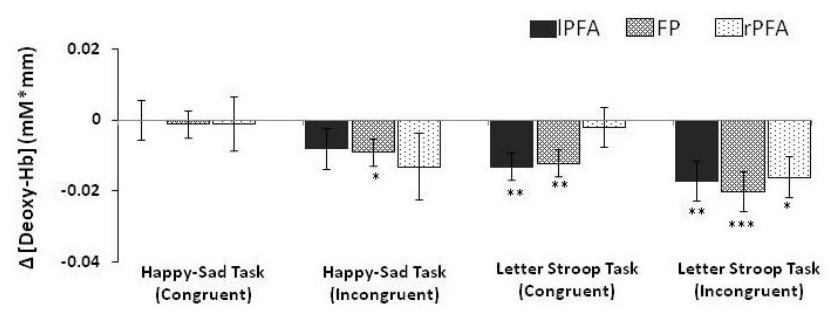

Figure 4. Change of deoxy-Hb concentration for each task and condition in each ROI

$* \mathrm{p}<0.05, * * \mathrm{p}<0.01, * * * \mathrm{p}<0.001$.

The mean time courses of oxy-Hb and deoxy-Hb concentrations during the incongruent condition in the Happy-Sad task and the Letter Stroop task are shown in Figure 5 and Figure 6.

\subsection{SCR Data}

Figure 7 shows changes (the common logarithm value) in the SCR. A two way ANOVA revealed significant main effects of task $(F(1,28)=4.50, p<.05)$ and condition $(F$ $(1,28)=6.51, p<.05)$, however, there was no interaction between task and condition.

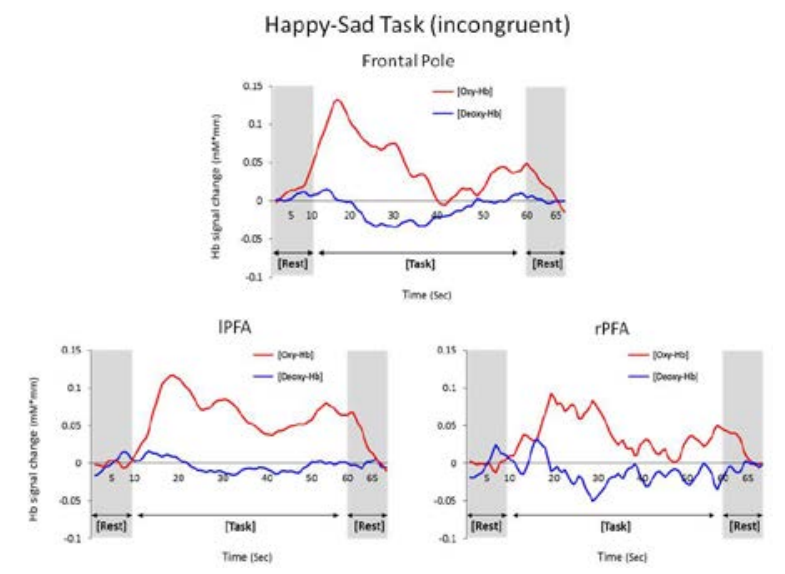

Figure 5. The mean time courses of oxy-Hb and deoxy-Hb concentration during the incongruent condition of the Happy-Sad task. 


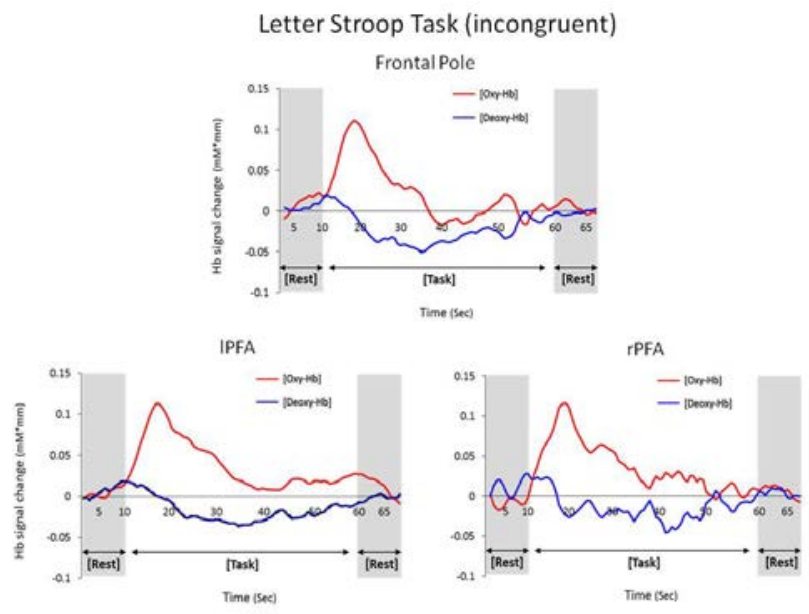

Figure 6. The mean time courses of oxy-Hb and deoxy-Hb concentration during the incongruent condition of the Letter Stroop task

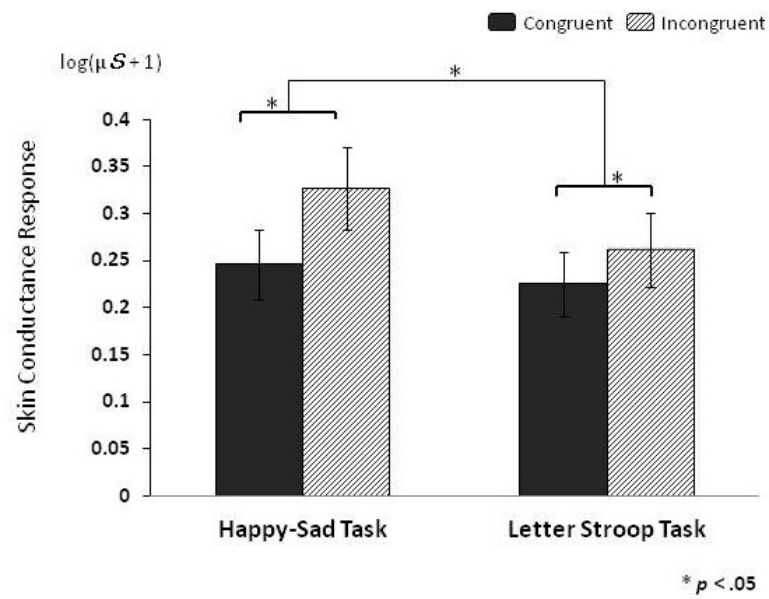

Figure 7. Change of skin conductance response during each condition of tasks

\section{Discussion}

Results of the present study suggest that the happy-sad task may have the same pattern of brain activation as the letter Stroop task. Especially, there were significant increases of oxy-Hb concentrations during the incongruent condition in both tasks, whereas there were significant decreases of deoxy-Hb concentrations in all ROIs, with the exception of the IPFA region. Significant increases of oxy-Hb concentration in the IPFA and rPFA corroborate previous NIRS studies reporting that Stroop tasks were related to bilateral IFG activation [16,18,19,20,21]. Therefore, our data is consistent with previous reports suggesting an association with response inhibition and activity of inferior prefrontal area [13,14,15,29,30,31]. The mean correct response ratios of the incongruent conditions in both tasks were lower than that of the congruent conditions in both tasks. In addition, the mean correct response ratio of the incongruent condition in the happy-sad task was lower than that in the letter Stroop task, but all of the mean correct response ratios were over 95\% and correct response may not be big issue here.

Change of oxy-Hb concentration in the lPFA of the congruent condition in the happy-sad task was significantly greater than that in the congruent condition in the letter Stroop task. The left inferior frontal region is known to be associated with recognition of facial expressions [32,33,34,35]. A NIRS study using face stimuli has reported a significant increase in the activity of the left prefrontal region in the sad and happy facial expression condition [36]. Change of oxy-Hb concentration in all ROIs did not differ significantly from the baseline for the congruent condition in the happy-sad task. One explanation for this finding could be that the large number of trials resulted in a habituation effect. Another possibility could be that the low cognitive load in the congruent condition decreased oxy-Hb activation. Ochsner et al [37] reported that top-down generation of emotions activated left prefrontal, cingulate, and temporal regions. The incongruent condition may have caused a higher load than the congruent condition because of cognitive reappraisal [38] and processing bias [14].

In the FP, changes of oxy-Hb concentration in the happy-sad task were significantly greater than that in the letter Stroop task, regardless of the condition. The FP region is known to activate when reflecting on one's own emotional states, as well as when ascribing mental states to other agents [39]. The activation peaks observed in this study were predominantly located in medial Brodmann's area 10 . However, the oxy-Hb activation in the FP may be associated with activities of above cognitive functions as indicated by Gilbert et al [39], because these activation peaks were extremely close to rostral PFA.

$\mathrm{Oxy}-\mathrm{Hb}$ in the IPFA and rPFA increased in the incongruent condition of both tasks. This result corroborates a meta-analysis of the Stroop Task conducted by Laird et al [13]. Especially, the right IFC is known to play an inhibitory role [15]. The incongruent condition in the happy-sad task and letter Stroop task was associated with inhibitory control. Thus, activation of rPFA observed in this study suggests that the participant inhibited inappropriate behaviors in the incongruent conditions.

SCR changes in the happy-sad task were greater than in the letter Stroop task. It is known that emotional suppression results in enhanced SCR [40]. Furthermore, the SCR value in the incongruent condition was greater than in the congruent condition. This result is consistent with a previous study [41], which reported that activity in lateral regions of prefrontal cortex reflects the rate of change in the SCR $[42,43,44]$. Our findings suggest that brain activities in the happy-sad task were associated with suppression of emotions and behavioral inhibition.

Several methodological limitations of this study however, should be noted. It is unlikely that the sourcedetector separation of $3 \mathrm{~cm}$ used in the current study could measure further than a depth of $1.5-2 \mathrm{~cm}$ below the scalp [45]. Thus, the NIRS could have detected hemoglobin changes only in the outer, approximately 5-10 mm of brain tissue. Brain activities in the medial prefrontal cortex, anterior cingulate cortex, orbitofrontal cortex, and other brain regions located more internally, that are also known to be related to cognitive and emotional functions could not be assessed in this study. Moreover, in order to avoid the emotional effects of time pressure on decisionmaking, we did not time each reaction during the task performance. Therefore, it was not possible to assess differences in cognitive processing based on reaction time during the two tasks. However, Ovaysikia et al [46] using emotional words and emotional faces in the Stroop task, reported that reaction times for the letter stimulus task was 
faster than that for the face stimulus task. It is suggested that future studies should investigate differences in reaction times between the happy-sad task and the letter Stroop task.

In conclusion, the present study examined differences in the activity of prefrontal cortex when conducting the happy-sad task and the letter Stroop task. Our results indicated that there was activation in brain regions during both tasks. Especially, the happy-sad task resulted in greater activation than the letter Stroop task in the incongruent condition. In addition, amplitude of the SCR for the happy-sad task was greater than that for the letter Stroop task. It is concluded that our findings suggest that the brain activities in the happy-sad task are associated with the suppression of emotions and the inhibition of behavior.

\section{Acknowledgement}

This study was supported by a Grant-in-Aid for Scientific Research (B) 20330141, 26285155, Grand-inAid for Exploratory Research 26590143 and Grant-in-Aid for Scientific Research on Innovative Areas 26118707 from the Japan Society for the Promotion of Science (JSPS).

\section{References}

[1] Miller, E. K. (2000). The prefrontal cortex and cognitive control. Annual review of neuroscience, 1, 59-65.

[2] Stroop, J. R. (1935). Studies of interference in serial verbal reactions. Journal of Experimental Psychology, 18, 643-662.

[3] Miller, E. K., \& Cohen, J. D. (2001). An integrative theory of prefrontal cortex function. Annual Review of Neuroscience, 24, 167-202.

[4] Comalli, P.E.Jr, Wapners, S., \& Werener,H. (1962). Interference effects of Stroop color-word test in childhood, adulthood, and aging. The Journal of Genetic Psychology, 100, 47-53.

[5] Charchat-Fichman, H., \& Oliveira, R. M. (2009). Performance of 119 Brazilian children on Stroop paradigm-Victoria version. Arquivos de Neuro-Psiquiatria, 67, 445-449.

[6] Gerstadt, C. L., Hong, Y. J., \& Diamond, A. (1994). The relationship between cognition and action: performance of children 3 1/2-7 years old on a Stroop-like day-night test. Cognition, 53, 129-153.

[7] Montgomery, D.E., \& Koeltzow, T.E. (2010). A review of the daynight task: The Stroop paradigm and interference control in young children. Developmental Review, 30, 308-330.

[8] Lagattuta, K. H., Sayfan, L., \& Monsour, M. (2011). A new measure for assessing executive function across a wide age range: children and adults find happy-sad more difficult than day-night. Developmental Science, 14, 481-489.

[9] Adleman, N.E., Menon, V., Blasey, C.M., White, C.D., Warsofsky, I.S., Glover, G.H., et al. (2002). A developmental fMRI study of the Stroop color-word task. Neuroimage, 16, 61-75.

[10] MacDonald, A.W., Cohen, J.D., Stenger, V.A., \& Carter, C.S. (2000). Dissociating the role of the dorsolateral prefrontal and anterior cingulate cortex in cognitive control. Science, 288, 18351838.

[11] Bench, C.J., Frith, C.D., Grasby, P.M., Friston, K.J., Paulesu, E., Frackowiak, R.S., et al. (1993). Investigations of the functional anatomy of attention using the Stroop test. Neuropsychologia, 31, 907-922.

[12] Peterson, B. S., Skudlarski, P., Gatenby, J. C., Zhang, H., Anderson, A. W., \& Gore, J. C. (1999). An fMRI study of Stroop word-color interference: Evidence for cingulate subregions subserving multiple distributed attentional systems. Biological Psychiatry, 45, 1237-1258.

[13] Laird, A.R., McMillan, K.M., Lancaster, J.L., Kochunov, P., Turkeltaub, P.E., Pardo, J.V., et al. (2005). A comparison of label- based review and ALE meta-analysis in the stroop task. Human Brain Mapping, 25, 6-21.

[14] Taylor, S.F., Kornblum, S., Lauber, E.J., Minoshima, S., \& Koeppe, R.A. (1997). Isolation of specific interference processing in the Stroop task: PET activation studies. Neuroimage, 6, 81-92.

[15] Aron, A.R., Robbins, T.W., \& Poldrack, R.A. (2004). Inhibition and the right inferior frontal cortex. Trends in Cognitive Sciences, 8, 170-177.

[16] Ehlis, A.C., Herrmann, M.J., Wagener, A., \& Fallgatter, A.J. (2005). Multi-channel near-infrared spectroscopy detects specific inferior-frontal activation during incongruent stroop trials. Biological Psychology, 69, 315-331.

[17] Hyodo, K., Dan, I., Suwabe, K., Kyutoku, Y., Yamada, Y., Akahori, M., et al. (2012). Acute moderate exercise enhances compensatory brain activation in older adults. Neurobiology of Aging, 33, 26212632.

[18] Léon-Carrion, J., Damas-López, J., Martín-Rodríguez, J. F., Domínguez-Roldán, J. M., Murillo-Cabezas F., Barroso Y Martin, J.M., et al. (2008). The hemodynamics of cognitive control: The level of concentration of oxygenated hemoglobin in the superior prefrontal cortex varies as a function of performance in a modified stroop task. Behavioural Brain Research, 193, 248-256.

[19] Schroeter, M.L., Zysset, S., Kruggel, F., \& von Cramon, D.Y. (2003). Age dependency of the hemodynamic response as measured by functional near-infrared spectroscopy. Neuroimage, $19,555-564$.

[20] Schroeter, M.L., Zysset, S., Wahl, M., \& von Cramon, D.Y. (2004). Prefrontal activation due to stroop interference increases during development - an event-related fNIRS study. Neuroimage, 23, 1317-1325.

[21] Taniguchi, K., Sumitani, S., Watanabe, Y., Akiyama, M., \& Ohmori, T. (2012). Multi-channel near-infrared spectroscopy reveals reduced prefrontal activation in schizophrenia patients during performance of the kana Stroop task. The journal of medical investigation, 59, 45-52.

[22] Koizumi, H., Yamamoto, T., Maki, A., Yamashita, Y., Sato, H., Kawaguchi, H., et al. (2003). Optical topography: practical problems and new applications. Applied Optics, 42, 3054-3062.

[23] Matsuoka, K., Masatake, U., Kasai, K., Koyama, K., \& Kim, Y. (2006). Estimation of premorbid IQ in individuals with Alzheimer's disease using Japanese ideographic script (Kanji) compound words: Japanese version of National Adult Reading Test. Psychiatry and Clinical Neurosciences, 60, 332-339.

[24] Suda, M., Fukuda, M., Sato, T., Iwata, S., Song, M., Kameyama, M., et al. (2009). Subjective feeling of psychological fatigue is related to decreased reactivity in ventrolateral prefrontal cortex. Brain Research, 1252, 152-160.

[25] Cadenhead, K.S., Perry, W., Shafer, K., \& Braff, D.L. (1999). Cognitive functions in schizotypal personality disorder. Schizophrenia Research, 37, 123-132.

[26] Kim, M-S., Oh, S.H., Jang, K.M., Che, H., \& Im, C-H. (2011). Electrophysiological correlates of cognitive inhibition in college students with schizotypal traits. Open Journal of Psychiatry, 2, 6876.

[27] Ito, S., Ohbe, S., Ohta, M., Takao, T., \& Sakamoto, S. (2008). The reliability and validity of the Japanese version of Schizotypal Personality Questionnaire Brief. Japanese Bulletin of Social Psychiatry, 17, 168-176. (In Japanese)

[28] Baumgartner, T., Esslen, M., Jäncke, L. (2006). From emotion perception to emotion experience: emotions evoked by pictures and classical music. International Journal of Psychophysiology, 60 34-43.

[29] Derrfuss, J., Brass, M., Neumann, J., \& von Cramon, D.Y. (2005) Involvement of the inferior frontal junction in cognitive control: meta-analyses of switching and Stroop studies. Human Brain Mapping, 25, 22-34.

[30] Floden, D., Vallesi, A., \& Stuss, D.T. (2011). Task context and frontal lobe activation in the Stroop task. Journal of Cognitive Neuroscience, 23, 867-879.

[31] Kemmotsu, N., Villalobos, M.E, Gaffrey, M.S., Courchesne, E., \& Müller R.A. (2005). Activity and functional connectivity of inferior frontal cortex associated with response conflict. Cognitive Brain Research, 24, 335-342.

[32] Chong, T.T., Williams, M.A., Cunnington, R., \& Mattingley, J.B. (2008). Selective attention modulates inferior frontal gyrus activity during action observation. Neuroimage, 40, 298-307. 
[33] Keuken, M.C., Hardie, A., Dorn, B.T., Dev, S., Paulus, M.P., Jonas, K.J., et al. (2011). The role of the left inferior frontal gyrus in social perception: an rTMS study. Brain research, 1383, 196-205.

[34] Pobric, G., \& Hamilton, A. F. (2006). Action understanding requires the left inferior frontal cortex. Current Biology, 16, 524529.

[35] Sprengelmeyer, R., Rausch, M., Eysel, U.T., \& Przuntek, H. (1998). Neural structures associated with recognition of facial expressions of basic emotions. Proceedings of Royal Society of London. Series B. Biological Sciences, 265, 1927-1931.

[36] Herrmann, M.J., Ehlis, A.C., \& Fallgatter, A.J. (2003). Prefrontal activation through task requirements of emotional induction measured with NIRS. Biological Psychology, 64, 255-263.

[37] Ochsner, K.N, Ray, R.R., Hughes, B., McRae, K., Cooper, J.C., Weber, J., et al. (2009). Bottom-up and top-down processes in emotion generation: common and distinct neural mechanisms. Psychological Science, 20, 1322-1331.

[38] Gross, J.J. (2002). Emotion regulation: affective, cognitive and social consequences. Psychophysiology, 39, 281-291.

[39] Gilbert, S.J., Spengler, S., Simons, J.S., Steele, J.D., Lawrie, S.M., Frith, C.D., et al. (2006). Functional specialization within rostral prefrontal cortex (area 10): a meta-analysis. Journal of Cognitive Neuroscience, 18, 932-948.
[40] Ohira, H., Nomura, M., Ichikawa, N., Isowa, T., Iidaka, T., Sato, A., et al. (2006). Association of neural and physiological responses during voluntary emotion suppression. Neuroimage, 29, 721-733.

[41] Kobayashi, N., Yoshino, A., Takahashi, Y., \& Nomura, S. (2007). Autonomic arousal in cognitive conflict resolution. Autonomic neuroscience, 132, 70-75.

[42] Nagai, Y., Critchley, H.D., Featherstone, E., Trimble, M R., \& Dolan, R.J. (2004). Activity in ventromedial prefrontal cortex covaries with sympathetic skin conductance level: a physiological account of a "default mode" of brain function. Neuroimage, 22, 243-251.

[43] Raine, A., Reynolds, G. P., \& Sheard, C. (1991). Neuroanatomical correlates of skin conductance orienting in normal humans: a magnetic resonance imaging study. Psychophysiology, 28, 548-558.

[44] Tranel, D., \& Damasio, H. (1994). Neuroanatomical correlates of electrodermal skin conductance responses. Psychophysiology, 31, 427-438.

[45] Fukui, Y., Ajichi, Y., \& Okada, E. (2003). Monte Carlo prediction of near-infrared light propagation in realistic adult and neonatal head models. Applied Optics, 42, 2881-2887.

[46] Ovaysikia, S., Tahir, K.A., Chan, J.L., \& DeSouza, J F. (2011). Word wins over face: emotional Stroop effect activates the frontal cortical network. Frontiers in Human Neuroscience, 4, Article 234. 